\title{
Basic Science Meets Clinical Research: 10th North American Symposium on Acupuncture
}

\section{Sheraton Fisherman's Wharf Hotel, San Francisco, USA, July 1, 2004}

\author{
Taras I. Usichenko ${ }^{1}$ and Sheng-Xing $\mathrm{Ma}^{2}$
}

${ }^{1}$ Department of Anesthesiology and Intensive Care Medicine, Ernst Moritz Arndt University, Greifswald, Germany and ${ }^{2}$ Department of Obstetrics and Gynecology, David Geffen School of Medicine at University of California Los Angeles, Harbor-UCLA Medical Center, USA

\section{General Organization}

The annual North American Symposium on Acupuncture is the main scientific part of the educational program in Traditional Chinese Medicine (TCM) established by the Academy of Pain Research in 1979. Dr T. N. Lee, Founder and Director of the Academy and distinguished researcher who proposed the Thalamic Neuron Theory to explain the analgesic effects of acupuncture (1), maintains the scientific and educational quality of the Symposium at a high level.

The program covers various subjects in the field of clinical application of acupuncture, including pain management, internal medicine, family practice, obstetrics and gynecology, pediatrics, orthopedics, emergency medicine, psychiatry and much more.

The Symposium focused primarily on body acupuncture, ear acupuncture and diagnostic techniques, with an emphasis on TCM concepts as well as a modern scientific approach.

Both modern basic and clinical sciences and TCM teachings should be integrated in order to improve the efficacy of acupuncture in modern medical practice. With this central purpose in mind, every year Dr Lee invites the leading experts in basic and clinical acupuncture research, most of whom hold professorships at prominent medical schools or are affiliated with excellent academic institutions.

\section{Scientific Reports}

\section{Basic Research on Acupuncture}

J.C. Longhurst and Peng Li (Department of Medicine, University of California Irvine) reported on their studies of

For reprints and all correspondence: Taras I. Usichenko.

E-mail: taras@uni-greifswald.de acupuncture on the regulation of the cardiovascular system. Dr Longhurst provided an update on his research into the mechanisms of acupuncture regulation of the cardiovascular system. His results demonstrate that electroacupuncture (EA) can reduce myocardial ischemia in an animal model of demand-induced ischemia, mainly through reducing the demand of the myocardium for oxygen, rather than by enhancing blood flow. He has also found that both unmyelinated (or group IV) sensory fibers and finely myelinated (or group III) fibers are responsible for the EA-cardiovascular effects. Dr Li reported on the effects of acupuncture on exercise-induced cardiovascular responses in hypertensive patients. They found that arterial blood pressure responses to exercise were reduced in $70.6 \%$ of their subjects following EA of either PC 5-6 or LI 4-L7 acupuncture points. EA administered 1-2 times per week for 2 weeks resulted in a decrease in systolic blood pressure of $\sim 20 \mathrm{mmHg}$ in patients with hypertension.

Sheng-Xing Ma (Harbor-UCLA Medical Center, David Geffen School of Medicine at the University of California Los Angeles) reported that L-arginine-derived nitric oxide (NO) in the gracile nucleus mediates cardiovascular and antinociceptive responses to EA stimulation of the ST 36 acupuncture point in rats. Results showed that EA of point ST 36 produced analgesia and decreased arterial blood pressure in rats. The analgesic and cardiovascular responses to EA were facilitated by L-arginine NO and blocked by inhibitors of NO synthesis in the gracile nucleus (2,3). Dr Ma found that EA stimulation of point ST 36 induced expression of neuronal NO synthase in the gracile nucleus and cFos expression in the gracile nucleus-thalamic nuclei-cortex pathways. Further, the excitabilities of EA-sensitive thalamic units identified in the ventroposterolateral and paraventricular thalamic nuclei were

The online version of this article has been published under an open access model. Users are entitled to use, reproduce, disseminate, or display the open access version of this article provided that: the original authorship is properly and fully attributed; the Journal and Oxford University Press are attributed as the original place of publication with the correct citation details given; if an article is subsequently reproduced or disseminated not in its entirety but only in part or as a derivative work this must be clearly indicated. 
modified by microinjections of L-arginine, an NO donor and a selective inhibitor of neuronal NO synthesis, into the gracile nucleus. These results represent a novel discovery of how stimulation by acupuncture may induce the important endogenous substance NO in the gracile nucleus, which plays an important role in pain homeostasis and nociceptive/cardiovascular regulation. This is the first discovery of an endogenous NOergic substance contributing to signal transduction of acupuncture information through dorsal medulla-thalamic pathways since the previous discovery of opioid peptidemediated EA effects (3).

\section{Presentation of Clinical Trials on the Efficacy of Acupuncture and Related Techniques}

Joseph Audette (Department of Physical Medicine and Rehabilitation, Harvard Medical School) discussed specific methodological problems in clinical research concerning the effectiveness of acupuncture. He reported promising results from rigorous randomized controlled studies (4) on the effectiveness of acupuncture in the treatment of myofascial pain and headache.

Taras Usichenko (Anesthesiology and Intensive Care Medicine Department, University of Greifswald, Germany) reported on pain and joint stiffness reduction in patients with rheumatoid arthritis, treated with low-intensity electromagnetic millimeter waves (MW) applied to acupuncture points (5). Dr Usichenko also presented the model of the 'electromagnetic frame' of the human body based on the principles of quantum physics and the data from embryological, physiological and clinical research, which allows us to interpret the nature of acupuncture meridians and, thus, to explain the mechanism of MW action (6).

Ragnhild Kinge (Department of Obstetrics, Oslo University Hospital, Norway) reported the results of the randomized non-blinded controlled study, where acupuncture reduced the requirement for meperidine and provided high patient satisfaction during labor (7).

\section{General Impressions}

The audience of the 10th North American Symposium on Acupuncture received updated information on recent advances in scientific and clinical research on acupuncture. Almost all medical practitioners had previous experience of acupuncture and were active participants in the discussion of scientific reports.

We believe that the 10th North American Symposium on Acupuncture was a successful step on the way to further integration of modern medical sciences with acupuncture diagnostics and therapeutics, and to the improvement in patient care and satisfaction. A further positive result of this meeting was that it provided a bridge between acupuncture mechanisms and their clinical, therapeutic effects. This may help to advance knowledge of acupuncture practice and enhance communication between research scientists and clinicians.

\section{References}

1. Lee TN. Thalamic Neuron Theory: Theoretical Basis for the Role Played by the Central Nervous System (CNS) in the Causes and Cures of All Diseases. Medical Hypotheses 1994;43:285-302.

2. Chen S, Ma SX. Nitric oxide on acupuncture (ST36)-induced depressor response in the gracile nucleus. J Neurophysiol 2003;90:780-5.

3. Ma SX. Neurobiology of acupuncture: Toward CAM. Evidence-based Complem Altern Med 2004;1:41-7.

4. Audette JF, Blinder RA. Acupuncture in the management of myofascial pain and headache. Curr Pain Headache Rep 2003;7:395-401.

5. Usichenko TI, Ivashkivsky OI, Gizhko VV. Treatment of rheumatoid arthritis with electromagnetic millimeter waves applied to acupuncture points-a randomized double blind clinical study. Acupunct Electrother Res 2003;28:11-8.

6. Sitko SP, Gizhko VV. Towards a quantum physics of the living state. J Biol Phys 1991;18:1-10.

7. Nesheim BI, Kinge R, Berg B, et al. Acupuncture during labor can reduce the use of meperidine: a controlled clinical study. Clin J Pain 2003;19:187-91. 


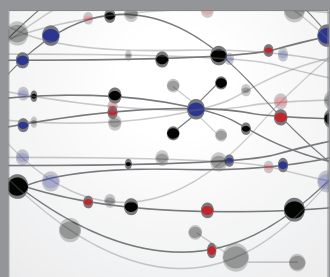

The Scientific World Journal
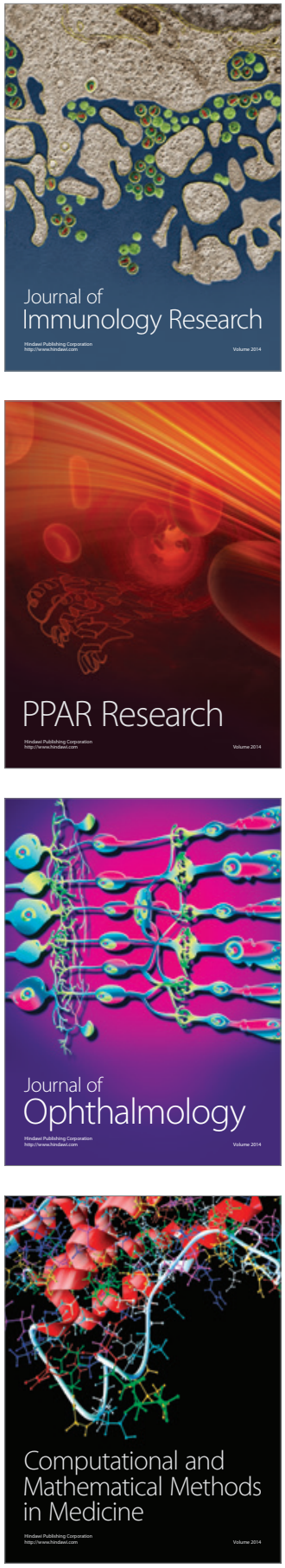

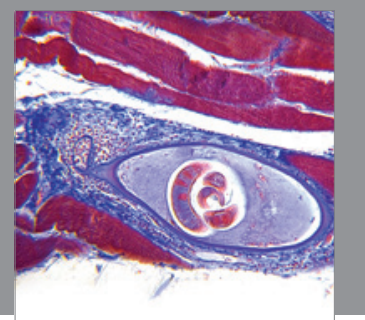

Gastroenterology

Research and Practice
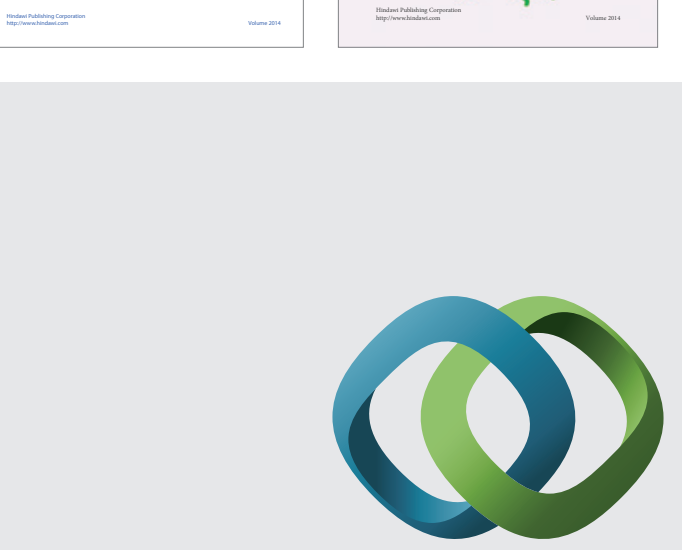

\section{Hindawi}

Submit your manuscripts at

http://www.hindawi.com
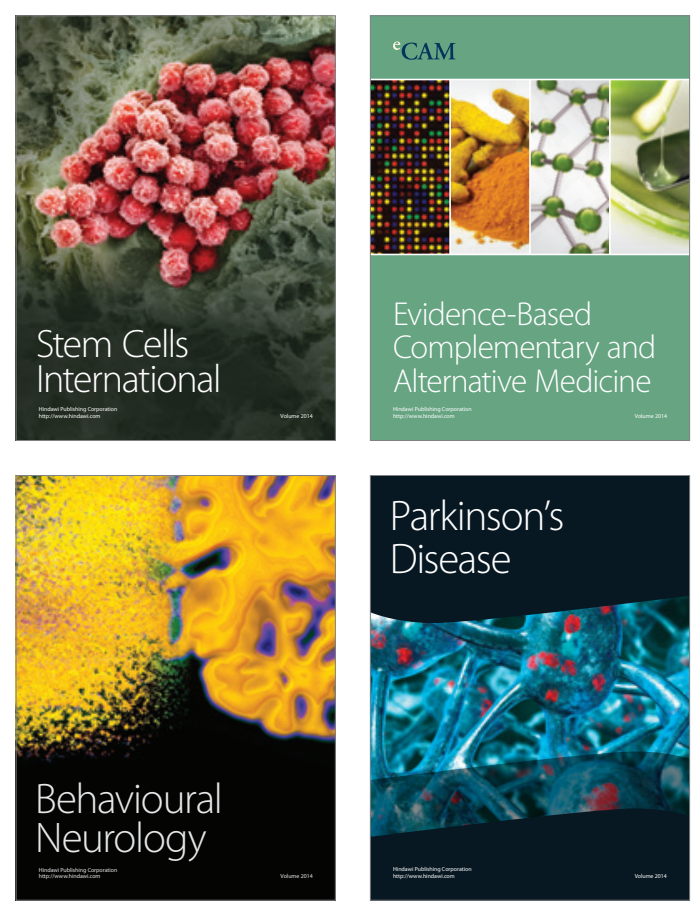

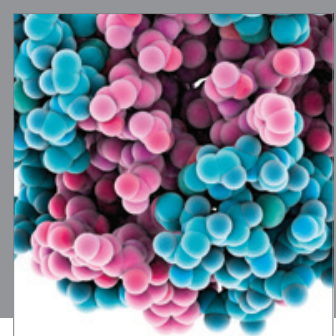

Journal of
Diabetes Research

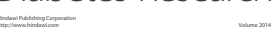

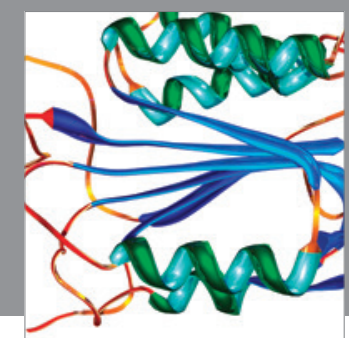

Disease Markers
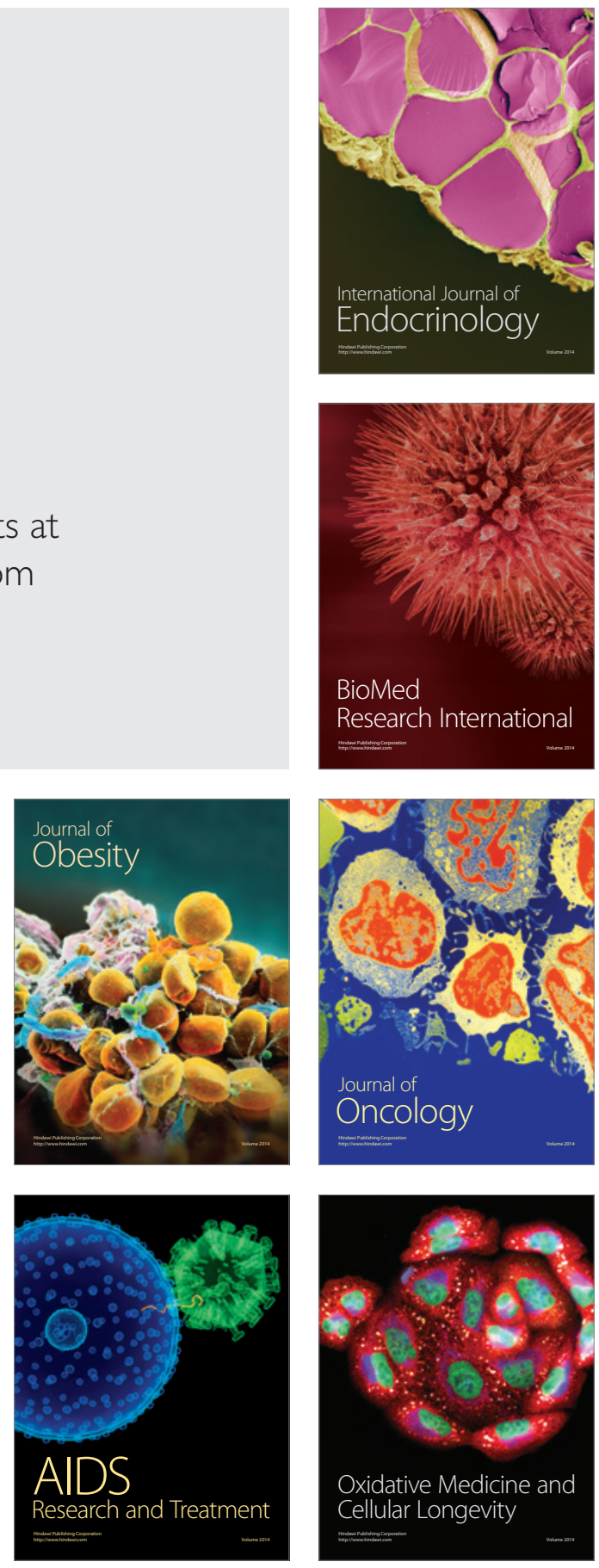